\title{
Correction of microtia with constriction features using a superficial temporal fascial flap combined with a rib cartilage graft
}

\author{
Joon Seok Lee, Jong Seong Kim, Jeong Woo Lee, Kang Young Choi, Jung Dug Yang, \\ Ho Yun Chung, Byung Chae Cho \\ Department of Plastic and Reconstructive Surgery, School of Medicine, Kyungpook National University, Daegu, Korea
}

\begin{abstract}
Background Microtia with constricted features is characterized by a short helical length of variable severity, upper antihelical or scaphal deficiency, and a downfolded upper ear. No consensus has been reached regarding the most appropriate surgical method for this condition. In this study, we aimed to introduce a simple and safe surgical method for the correction or reconstruction of upper helix ear deformities.

Methods Between February 2011 and June 2014, eight patients with microtia with constricted upper helix ear deformity underwent reconstruction of the ear deformity. The upper ear helical framework was constructed by carving and curving the eighth rib cartilage harvested from the ipsilateral chest wall, covering this cartilage with a superficial temporal fascial flap, and adjusting the skin graft to align with the ear contour. To evaluate their satisfaction, patients were asked to complete a questionnaire regarding ear shape, symmetry, position, color, and overall outcome scored on a 5-point scale at 12 months postoperatively.

Results None of the patients experienced severe complications in the reconstructed ear. The preoperative and postoperative vertical ear length ratios were 0.88 and 1.02, respectively. And the mean patient satisfaction scores for shape, symmetry, position, color, and overall outcome were $4.2,4.5,4.7,4.4$, and 4.6 out of 5 points, respectively. All patients expressed a high level of satisfaction at 12 months postoperatively.

Conclusions Our technique provides a good alternative method for the reconstruction of moderate constricted upper helix ear deformities in patients who meet the surgical indications with satisfactory outcomes and few complications.
\end{abstract}

Keywords Ears prominent and constricted / Congenital microtia / Ear auricle
Correspondence: Byung Chae Cho Department of Plastic and Reconstructive Surgery, School of Medicine, Kyungpook National University, 807 Hoguk-ro, Buk-gu, Daegu 41404, Korea

Tel: $+82-53-420-5688$

Fax: $+82-53-425-3879$

E-mail: medps@knu.ac.kr

Received: October 4, $2018 \bullet$ Revised: March 20, $2020 \bullet$ Accepted: March 20, 2020

pISSN: 2234-6163 • elSSN: 2234-6171 • https://doi.org/10.5999/aps.2018.01165• Arch Plast Surg 2020;47:317-323

This research was supported by the Kyungpook National University Research Fund in 2018.

\section{INTRODUCTION}

The global prevalence of ear deformities is approximately $5 \%$ [1]. Of the various types of ear deformities, defects in the upper third of the auricular cartilage manifest along a spectrum of severity, ranging from a mildly downfolded shape to microtia with constricted upper helix deformity. These latter defects are characterized by a short helical length and upper antihelical and

Copyright $(\odot) 2020$ The Korean Society of Plastic and Reconstructive Surgeons

This is an Open Access article distributed under the terms of the Creative Commons Attribution Non-Commercial License (https://creativecommons.org/

licenses/by-nc/4.0/) which permits unrestricted non-commercial use, distribution, and reproduction in any medium, provided the original work is properly cited.

www.e-aps.org 
scaphal deficiencies and require supplemental skin for correction. Although various surgical correction methods have been reported as ways to resolve these deformities [2-14], no consensus has been reached regarding the most appropriate method. As the severity of a constricted ear deformity and the shape of the intact ear vary among individuals, diverse approaches to reconstruction that consider both the ear position and shape are needed to achieve symmetry. Generally, the deformity in the upper helix is reconstructed, and preservation of the intact lower three-quarters of the ear is accepted as a standard technique to reduce discomfort and increase satisfaction. According to the report of Siegert and Magritz [15] regarding the reconstruction of peripheral ear deformities, the cartilage can be harvested up to $2.5 \mathrm{~cm}$ from the concha cavum for small defects, while surgery should be conducted in three stages for large defects. Park [7] reported a surgical procedure in which a difference in height of $1.5-2 \mathrm{~cm}$ between the normal and abnormal ear was categorized as belonging to Tanzer's group IIb. However, reports have not yet adequately addressed microtia with constricted upper helix deformity, in which cartilage coverage is difficult due to the presence of insufficient ear cartilage for the required extension of more than $1 \mathrm{~cm}$, even though the difference in height between both ears is relatively slight, at $1-1.5 \mathrm{~cm}$.

In the present study, we aimed to introduce a safe and reliable surgical method for the correction of microtia with constricted upper helix deformity based on a superficial temporal fascial flap that can be readily applied by plastic surgeons. We applied this one-stage technique to patients with microtia with constricted upper helix deformity who presented with a helical height difference of $1-1.5 \mathrm{~cm}$ between the normal and constricted ears and thus required supplemental skin for reconstruction.

\section{METHODS}

\section{Patient selection}

Patients with microtia with constricted upper helix deformity who underwent surgery between February 2014 and June 2018 were enrolled in this study (Table 1). Among patients without a surgical history, those with microtia with constricted upper helix deformity and a helical height difference of $1-1.5 \mathrm{~cm}$ between the normal and constricted ears were included and underwent reconstruction using our surgical method (Fig. 1). The surgical method was determined based on a preoperative gross ear deformity assessment and photometry. Patients with microtia without constricted features were excluded, because those deformities were equivalent to more standard cases of microtia (Fig. 2).

\section{Surgical procedures \\ Harvest of costal cartilage}

For this procedure, the eighth rib cartilage was split from the ipsilateral chest wall and harvested together with the perichondrium. First, the xiphoid process and the eighth (i.e., floating) rib were marked on the ipsilateral chest wall, and an incision was made through the eighth intercostal space to split the rectus abdominis muscle and expose the eighth rib. A periosteal elevator was used for dissection, starting from the intercostal muscle near the costal cartilage. A costal cartilage block was obtained, while taking care to avoid pleural injury. After using electrocautery to thoroughly control bleeding at the donor site, a $200-\mathrm{mL}$ negative pressure drain was inserted, and the rectus abdominis muscle, fascia, subcutaneous tissue, and skin were sutured using 3-0, 4-0 Vicryl, and 5-0 nylon sutures.

\section{Preparation of the ear deformity}

On the ear, a full-release incision was made to the concha area according to the normal ear framework. Once the incision was released to achieve a normal ear size, a subcutaneous pocket was dissected to $2-3 \mathrm{~cm}$ in the helical root and $3-4 \mathrm{~cm}$ in the midportion of the auricle to accommodate the fabricated helical cartilage. This fabrication was carved from the eighth rib cartilage using a no. 15 blade according to an X-ray image of the nor-

\section{Table 1. Patient characteristics}

\begin{tabular}{rcccccc}
\hline $\begin{array}{c}\text { Patient } \\
\text { No. }\end{array}$ & Age $(\mathrm{yr})$ & Sex & $\begin{array}{c}\text { Deformity } \\
\text { direction }\end{array}$ & $\begin{array}{c}\text { Preoperative } \\
\text { vertical ear length ratio }\end{array}$ & $\begin{array}{c}\text { Postoperative } \\
\text { vertical ear length ratio }\end{array}$ & $\begin{array}{c}\text { Vertical ear length (mm), } \\
\text { right/left }\end{array}$ \\
\hline 1 & 9 & Female & Left & 0.87 & 1.05 & $54 / 47$ \\
2 & 19 & Male & Right & 0.86 & 1.02 & $53 / 62$ \\
3 & 16 & Male & Left & 0.91 & 0.98 & $58 / 53$ \\
4 & 16 & Male & Right & 0.85 & 1.10 & $50 / 59$ \\
5 & 17 & Male & Left & 0.88 & 1.04 & $62 / 55$ \\
6 & 15 & Male & Left & 0.89 & 0.97 & $60 / 53$ \\
7 & 11 & Male & Left & 0.90 & 0.98 & $60 / 54$ \\
8 & 22 & Male & Left & 0.88 & 1.03 & $64 / 56$ \\
\hline
\end{tabular}




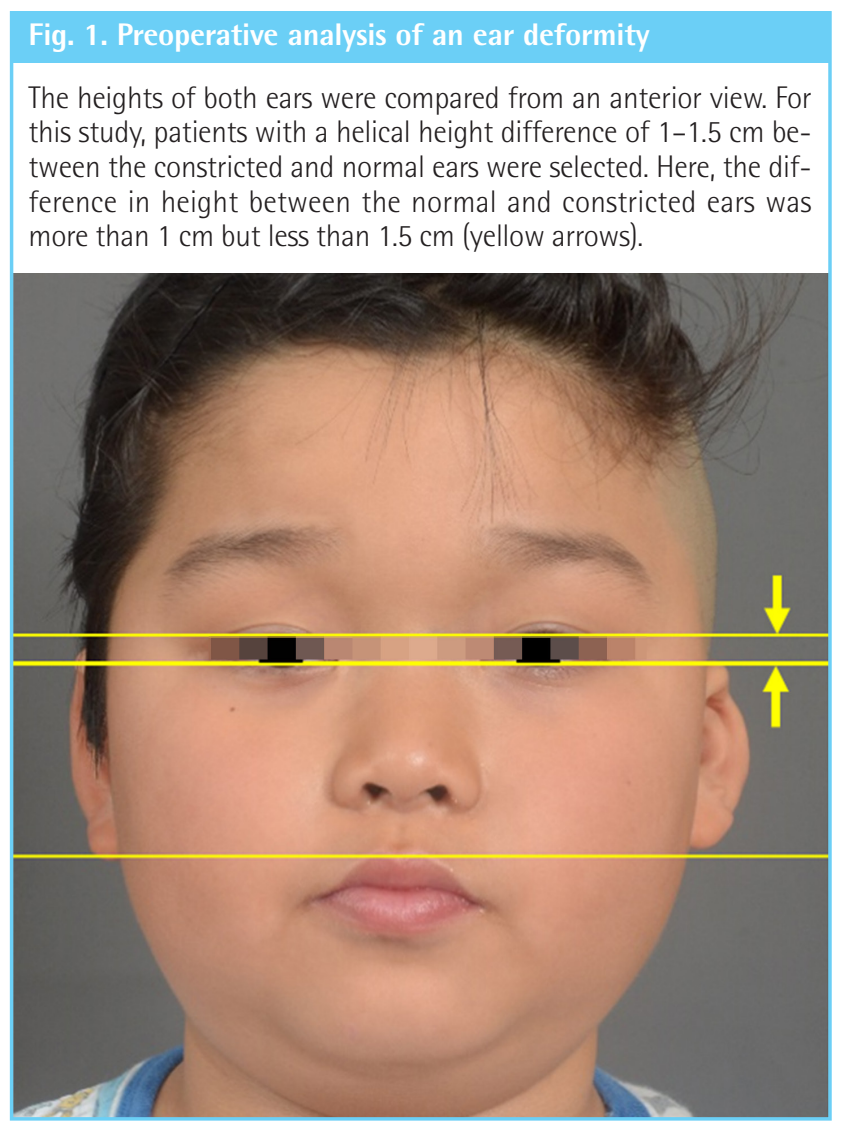

\section{Fig. 2. Microtia with constriction features}

The lower two-thirds of the ear was intact. However, supplemental skin was required in the upper ear helical area. (A) Anterior view and (B) oblique view images.

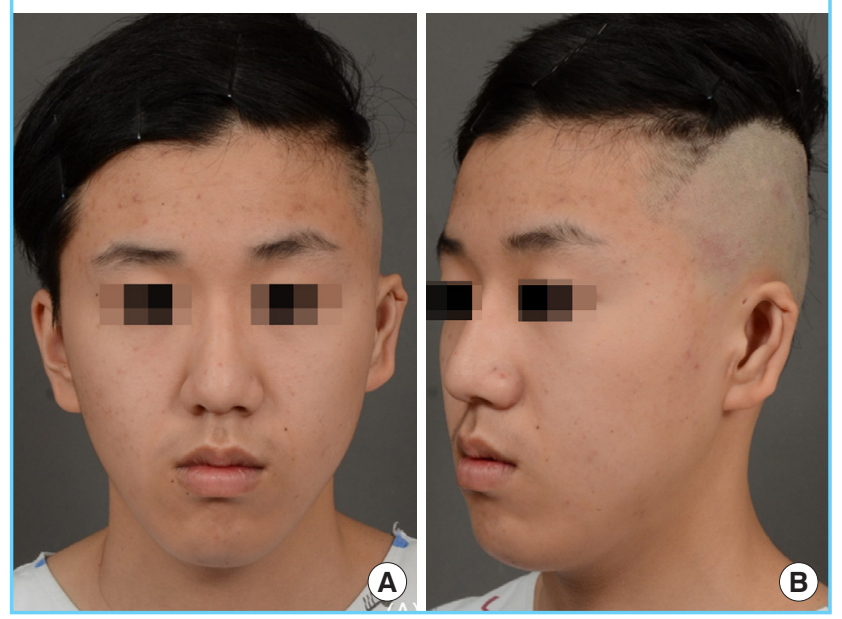

mal ear framework. The fabricated helical cartilage was cut to a length of 7-8 cm and inserted from the helical root to $3 \mathrm{~cm}$ within the subcutaneous pocket at the mid-portion of the auricle, with a height of 4-5 mm, a width of 3-4 mm width, and tails at the helical root and mid-portion of the helix. This fabricated helical cartilage was usually well curved and flexible, al-

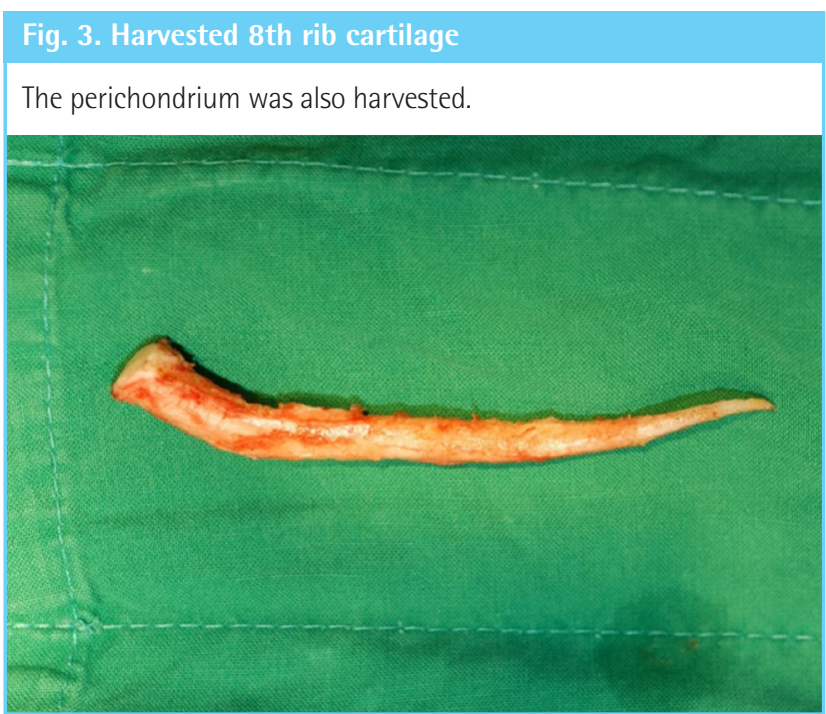

though stiffness due to calcification could be resolved by superficially scoring the outer surface of the fabricated cartilage in several places to prevent fracture and deformation. The fabricated helical cartilage was then inserted into the skin pockets of the helical root and the mid-portion of the helix, fixed to the original ear cartilage using a 4-0 white nylon suture in a position slightly higher than the antihelix in the upper third, and tilted outward by $10^{\circ}-15^{\circ}$ in the middle third. A blade was used to carve and narrow the outer portion of the helix. If the superior portion of the antihelix was also deficient, additional remnant cartilage was carved and fixed to the ear cartilage using a 5-0 nylon suture. The mean length was $5-6 \mathrm{~cm}$ (Fig. 3).

\section{Temporal fascial flap and split-thickness skin graft}

To generate a superficial temporal fascial flap, the hair was shaved around an $8-\mathrm{cm}$ radius from the auricle, and the incision line was chosen after confirming the location of the superficial temporal artery and the pedicle via Doppler ultrasonography (ES-100VX MiniDoppler Vascular Ultrasound Doppler; Koven Technology, Inc., Winnipeg, MB, Canada) (Fig. 4). First, to determine the amount of skin needed to cover the ear helix, the deformed ear was compared with the intact ear, and an incision was made in the area requiring correction to expose the cartilage. Next, the framework was formed by carving and designing the helical cartilage from the harvested rib cartilage, followed by insertion into the skin pockets as described above (Fig. 4). After measuring the positions, shapes, and sizes of both ears to determine the required amount of coverage, the superficial temporal fascial flap was incised and elevated carefully to avoid damaging the pedicle. After confirming that it was intact, tunneling was performed by turning over the flap beneath the skin in the ear helix area to cover the reconstructed cartilage. Using a feeding 


\section{Fig. 4. Reconstructed upper helical framework}

The incision was designed after marking the superficial temporal artery (i.e., the pedicle of the superficial temporal fascial flap) using Doppler ultrasonography. The black arrow indicates the superficial temporal fascial flap (pedicle), while the yellow arrow indicates the incision line designed around the pedicle. The white arrowheads point to the reconstructed upper helical framework composed of cartilage from the eighth rib.

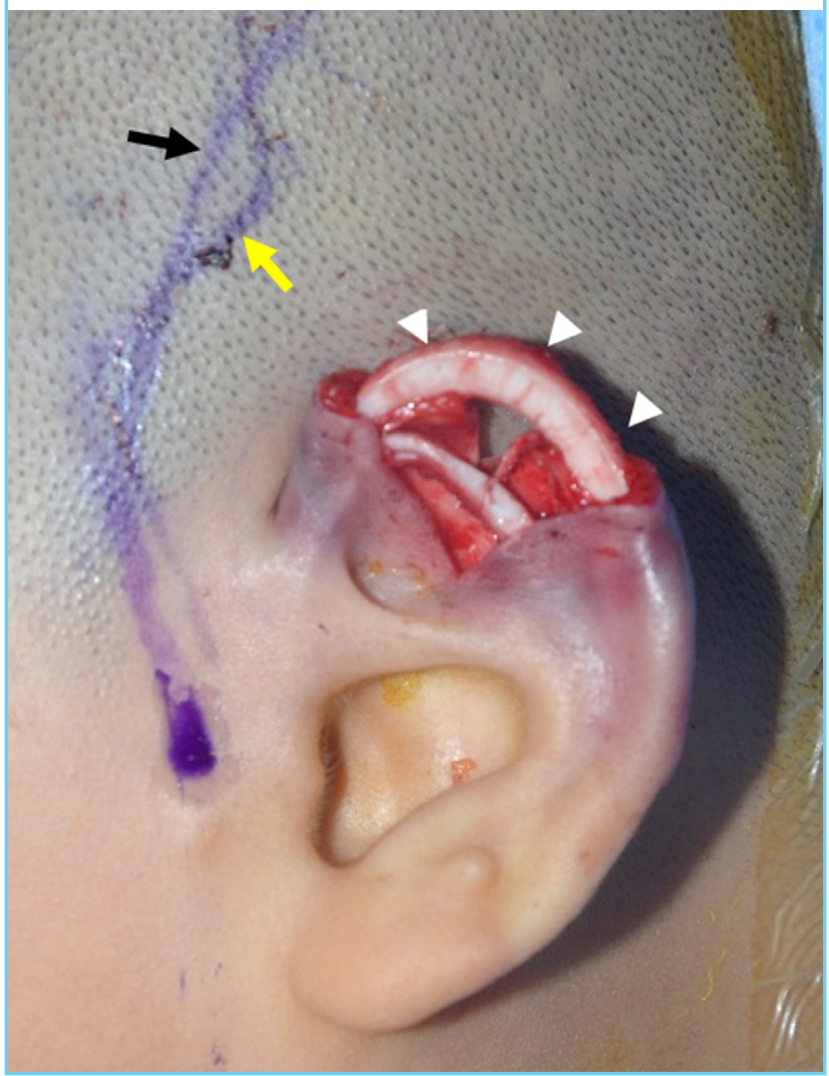

tube, a negative suction drain was placed inferior to the flap to minimize any adverse effects caused by hematoma or fluid collection. Next, quilting suturing was performed carefully using an absorbable subcuticular 5-0 Vicryl suture (Ethicon, Somerville, NJ, USA). After achieving convolution, a split-thickness skin graft was harvested from the ipsilateral inner arm and implanted. After comparing the convolution and shapes of both ears, a nopressure dressing was applied to conclude the surgery (Fig. 5).

\section{Postoperative evaluations}

Photoshop (Adobe, San Jose, CA, USA) was used to evaluate the degree of correction based on the vertical length ratio of the normal and constricted ears in preoperative and postoperative anterior view images (Table 1). To evaluate satisfaction after 12 months, patients completed a questionnaire intended to evaluate shape, symmetry, position, color, and overall outcome (Table 2). The items were scored on a 5-point scale, where scores of $1,2,3,4$, and 5 were assigned for the responses of very dissatisfied, dissatisfied, neutral, satisfied and very satisfied, respectively.

\begin{tabular}{|lc|}
\hline Table 2. Patient satisfaction \\
\hline Category & Score, mean \pm SD \\
\hline Shape & $4.2 \pm 0.4$ \\
Symmetry & $4.5 \pm 0.3$ \\
Position & $4.7 \pm 0.6$ \\
Color & $4.4 \pm 0.4$ \\
Overall outcome & $4.6 \pm 0.5$ \\
\hline
\end{tabular}

Items were scored on a 5-point scale of 1 (very dissatisfied), 2 (dissatisfied), 3 (neutral), 4 (satisfied), or 5 (very satisfied).

\section{Fig. 5. Superficial temporal fascial flap}

(A) A superficial temporal fascial flap was elevated carefully to avoid damaging the pedicle and tunneled to enable movement to the reconstructed ear helix. (B) Here, the rib cartilage framework is properly covered. (C) To minimize problems caused by fluid collection (hematoma or seroma), a negative drain was placed inferiorly to the flap using a feeding tube. (D) The donor site in the scalp was repaired, and skin grafting was performed on the vascularized superficial temporal fascial flap to match the contour of the ear helix.
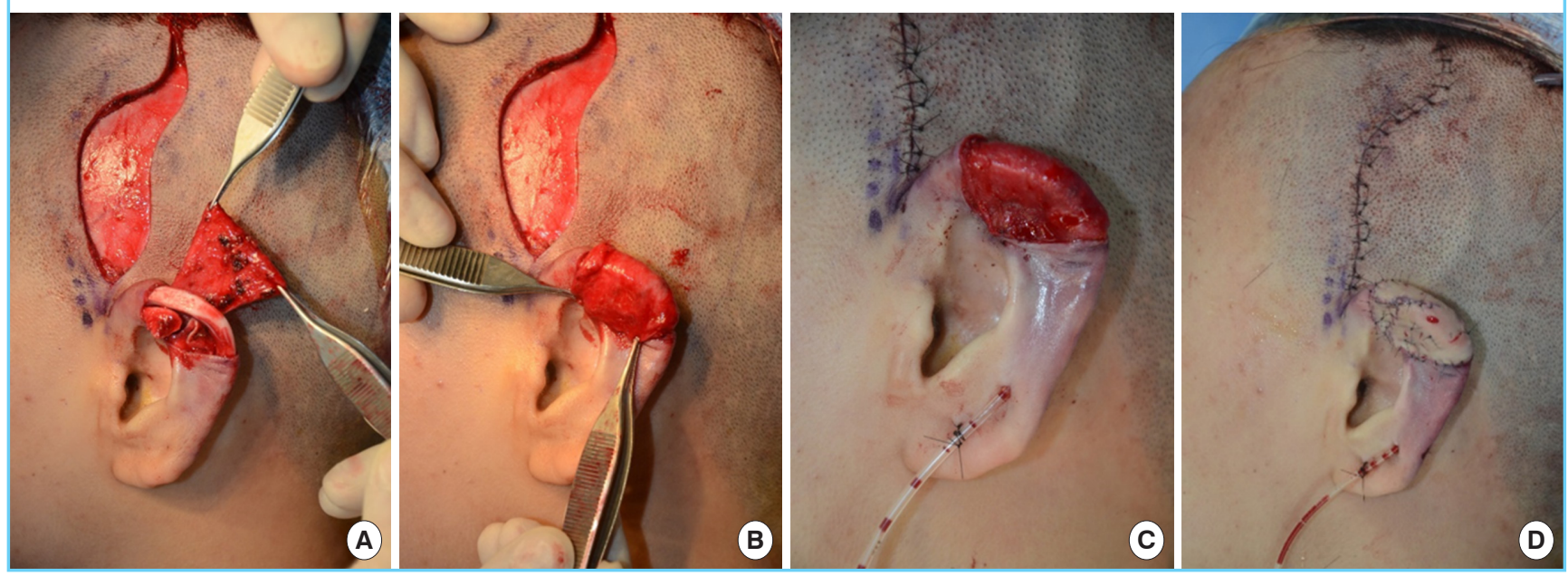


\section{RESULTS}

Of the 28 patients with constricted ear deformities, eight patients with microtia with unilateral constricted upper helix deformity (one female patient and seven male patients) were included in this study. The patients' ages ranged from 9 to 22 years. Six patients had a left constricted ear deformity, while two had a right constricted ear deformity. No patients experienced severe complications due to loss of the flap or skin graft. No problems with congestion were reported. One patient developed linear necrosis along the margin of the skin graft site, which resolved through conservative treatment (i.e., dressing) and did not require surgery. None of the patients experienced a severe complication at the rib cartilage donor site.

Despite initial complaints of color mismatches at the skin graft sites, the patients were satisfied with the reconstructed ear helices after 3 months. The incision lines in the superficial temporal fascia donor site were on the scalp and covered with hair. Therefore, no hair loss was visible and the patients did not complain of unsightly scars in this area. At the 12-month postoperative follow-up, the reconstructed ears had been maintained without any problems, and scar formation from the skin graft had improved. Moreover, the overall patient satisfaction level was reliable, with mean scores of $4.2 \pm 0.4,4.5 \pm 0.3,4.7 \pm 0.6,4.4 \pm 0.4$, and $4.6 \pm 0.5$ out of 5 points for shape, symmetry, position, color, and overall outcome, respectively. The preoperative and postoperative vertical ear length ratios were 0.88 and 1.02 , respectively, which confirmed the correction of the vertical ear length.

\section{DISCUSSION}

In this study, we introduced a safe, simple, and useful surgical method for the correction and restoration of constricted upper helix deformities. This method can be applied to a relatively wide area of the ear and in our study, yielded satisfactory outcomes with few complications.

Tanzer [2] defined a constricted ear as an ear deformity in which the helix appears to be tightened by a purse string. Cosman classified these deformities into four types according to clinical appearance, namely: lidding, protrusion, and decreased ear size. According to Janz et al. [1], most mild cases improve naturally with growth or can be resolved using a simple ear splinting method. Mohammadi et al. [16] and Woo et al. [17] reported that in neonates, non-surgical management (e.g., the molding method) is possible. However, moderately or more severely constricted ear deformities require surgical correction. Generally, a unilateral deformity is corrected by complementing the inadequate helix of the constricted ear with a cartilage framework that is subsequently covered by surrounding supplementary soft tissues. During this process, however, the amount of soft tissue may be insufficient to match the height of the intact ear. Accordingly, the helix framework can be complemented with rib cartilage and covered with a skin graft. Generally, the difference in height between the deformed and normal ear is approximately $1.0 \mathrm{~cm}$, but may exceed $3 \mathrm{~cm}$ in rare cases. In such cases, a pedicled flap, as opposed to a random flap, the superficial temporal fascia is elevated depending on the size of the defect, and the flap size may range from $1 \times 2 \mathrm{~cm}$ to $2 \times 3 \mathrm{~cm}$. The flap is turned over, and the skin is moved by undermining and tunneling. After suturing such that the convolutions in both ears are matched, the ear helical contour is matched by grafting splitthickness skin onto the superficial temporal fascial flap (Fig. 5).

Plastic surgeons are familiar with the anatomical features of the superficial temporal fascia and surrounding tissues. Therefore, it is not difficult to elevate a flap from this area. Furthermore, a superficial temporal fascial flap is not a random flap, but rather has a firm single pedicle. As previously described, it is difficult to elevate the position of a low-set ear deformity by using a local random flap of supplementary soft tissues around the auricle to cover the cartilaginous framework, as this process frequently leads to postoperative complications caused by congestion. Accordingly, the surgical technique described in this report appears to overcome these shortcomings in a reliable way. Although the height difference between the normal and abnormal ears in these patients is mild compared to those belonging to Tanzer group IIb, the difference should be corrected. These deformities are a very reliable indication for the one-stage operation for an extension of the upper helix by one-third.

For mild deformities with an ear height difference of $<1 \mathrm{~cm}$, the cartilage-reconstructed helix may be covered via V-Y advancement from the posterior auricular area, as reported by Ono et al. [18]. However, the range of the area that can be covered surgically and the extent of improvement are limited when using that approach. In contrast, our new technique adequately addresses these issues. In our method, cartilage from the eighth rib is used to supplement and reconstruct the defective helix. This donor tissue is widely used in other plastic surgical procedures (e.g., rhinoplasty, microtia ear reconstruction, and skull reconstruction) because it does not lead to complications. Further, we selected a pedicled flap to avoid severe congestion.

The method developed by Nagata can only correct the upper helical area of a constricted ear, while the method reported by Fisher et al. requires a total framework reconstruction after removing all ear cartilage $[19,20]$. Both methods are invasive and associated with too limited or too broad correction. Therefore, we developed the present surgical technique to correct low-set 


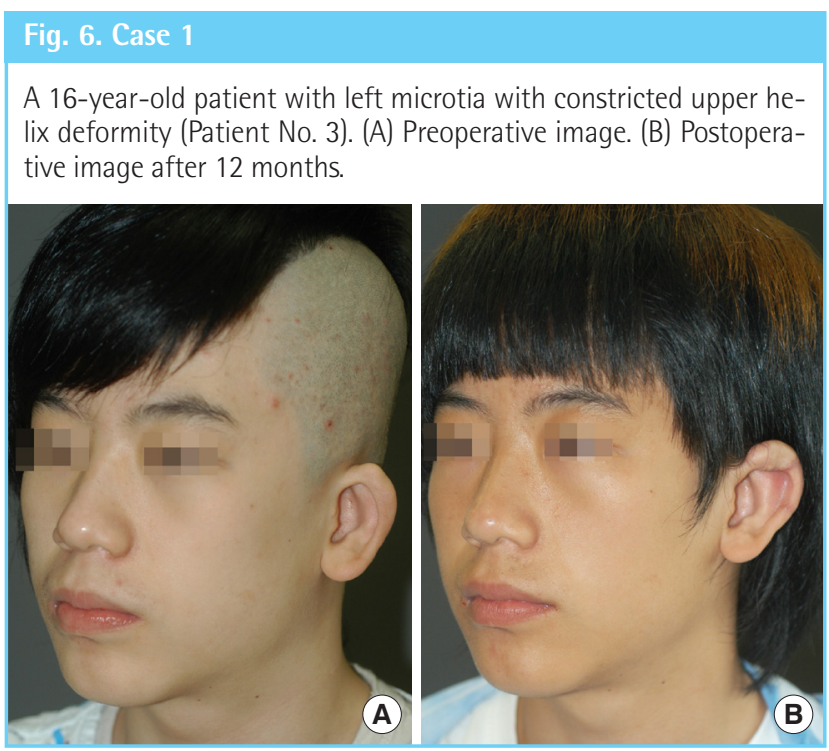

\section{Fig. 7. Case 2}

A 17-year-old patient with left microtia with constricted upper helix deformity (Patient No. 5). (A) Preoperative image. (B) Postoperative image after 6 years.
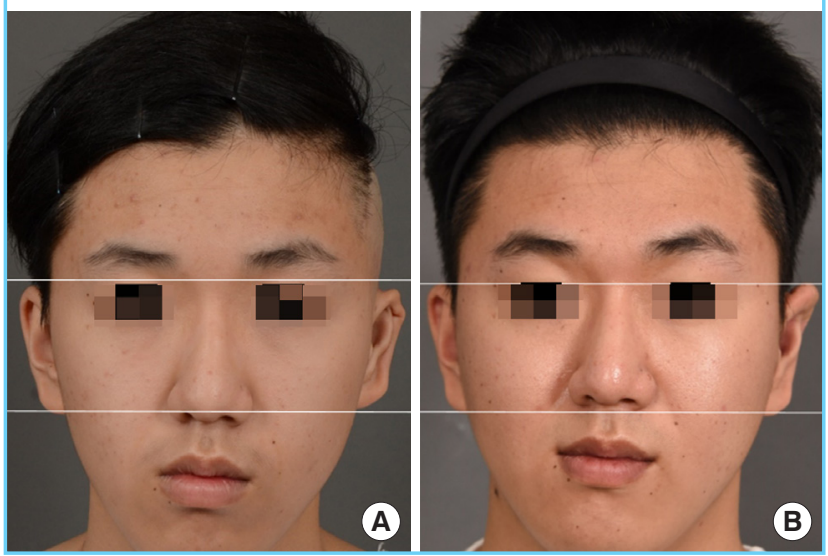

ears in various directions, using varied incisions. This technique is both less invasive and more efficient and reliable, as it preserves the normal ear framework in the lower three-quarters of the ear (Figs. 6, 7). Furthermore, our technique can produce good surgical outcomes in traumatic cases wherein one-third of the upper helix has been lost, a condition equivalent to microtia with constricted features (Fig. 8). In our sample, ears reconstructed using our technique showed good outcomes, with no deformation during the first postoperative year.

Comparisons of the degree of correction based on the vertical length ratios of the constricted and normal ears revealed that an ear with an average constriction of $88 \%$ was corrected to a ratio of nearly 1:1.02. Given the inherent limitations with regard to accurate measurements of ear length, as well as the importance

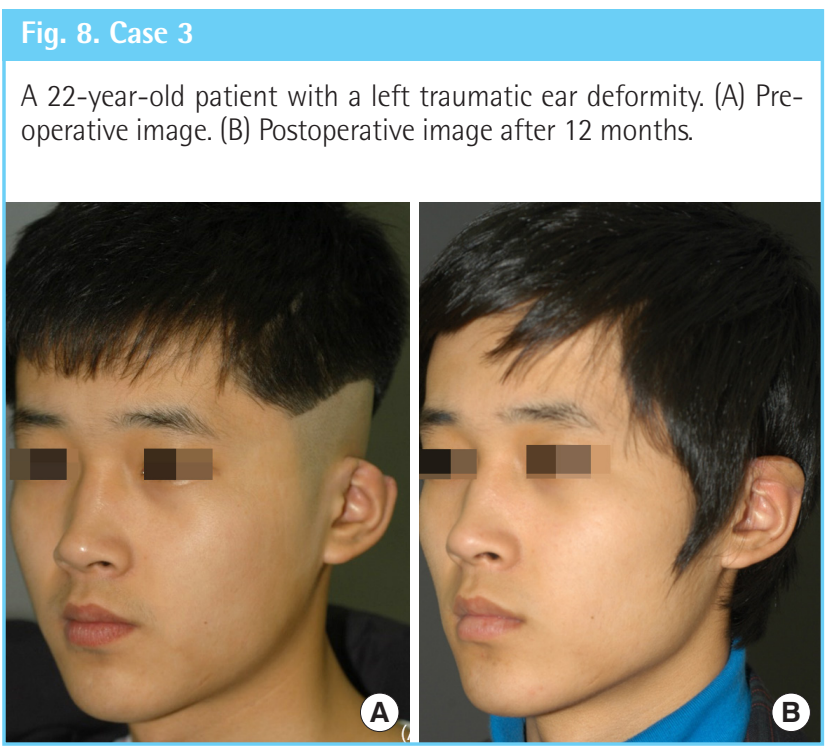

of the postoperative relationship of the corrected and normal ears for the surgical outcome of this procedure, we believe that comparing the ratio between the ears based on photos taken under uniform conditions provides a good and objective determination of the degree of correction. Moreover, the vertical ear length correction ratio provides an objective indicator of deviations over time that can be applied during long-term follow-up (Fig. 7). Moreover, a subjective satisfaction survey administered at the 12-month follow-up yielded a very high score (4.6 of 5) for the overall outcome, indicating a high level of patient satisfaction. Particularly high scores ( 4.7 and 4.5 points) were given for position and symmetry, respectively, which indicated that our method significantly corrected the low-set positions of the constricted ears.

Despite these successes, however, the study also had several limitations. First, a large incision must be made at the donor site on the scalp, although the scar may be covered with hair. A second scar must also be created in the chest wall. Second, this study had a small sample size. Third, despite the single-stage technique, reconstruction with an auricular area flap led to color mismatches. Appropriate patient selection is necessary. Although the patient questionnaire survey showed that color mismatches made some progress towards an acceptable level after some time, we consider it necessary to conduct continued research in the future.

In conclusion, our useful procedure combining a superficial temporal fascial flap with a rib cartilage graft is a safe and reliable alternative technique for the correction of microtia with constricted upper helix deformity. Our technique can be used to match not only the ear position, but also the ear shape and symmetry, with few complications. 


\section{NOTES}

\section{Conflict of interest}

No potential conflict of interest relevant to this article was reported.

\section{Ethical approval}

The study was approved by the Institutional Review Board of Kyungpook National University Chilgok Hospital (IRB No. KNUCH 2020-05-019) and performed in accordance with the principles of the Declaration of Helsinki. Written informed consents were obtained.

\section{Patient consent}

The patients provided written informed consent for the publication and the use of their images.

\section{Author contribution}

Conceptualization: BC Cho. Data curation: JS Lee, JS Kim. Formal analysis: JS Lee, JW Lee. Methodology: JS Lee, KY Choi. Project administration: HY Chung. Visualization: JD Yang. Writing - original draft: JS Lee, JS Kim. Writing - review \& editing: KY Choi, BC Cho. Approval of final manuscript: all authors.

\section{ORCID}

Joon Seok Lee

https://orcid.org/0000-0002-1580-0487

Jong Seong Kim

Jeong Woo Lee https://orcid.org/0000-0002-4725-9277 https://orcid.org/0000-0003-4903-6066

Kang Young Choi

Jung Dug Yang

Ho Yun Chung

Byung Chae Cho constr Surg 1980;66:13-21.

6. Horlock N, Grobbelaar AO, Gault DT. 5-Year series of constricted (lop and cup) ear corrections: development of the mastoid hitch as an adjunctive technique. Plast Reconstr Surg 1998;102:2325-35.

7. Park C. A new corrective method for the Tanzer's group IIB constricted ear: helical expansion using a free-floating costal cartilage. Plast Reconstr Surg 2009;123:1209-19.

8. Egemen O, Ozkaya O, Barutca SA, et al. Tanzer group IIB constricted ear repair with helical advancement and superior auricular artery chondrocutaneous flap. J Craniofac Surg 2012;23:728-31.

9. Bi Y, Lin L, Yang Q, et al. Surgical correction of constricted ear combined with Stahl's ear. J Plast Reconstr Aesthet Surg 2015;68:895-901.

10. Kim YS, Chung S. A simplified method for correcting Tanzer's group II constricted ears: construction of the superior crus as a strut with cartilage expansion grafting. J Plast Reconstr Aesthet Surg 2016;69:e71-6.

11. Park C, Park JY. Classification and algorithmic management of constricted ears: a 22-year experience. Plast Reconstr Surg 2016;137:1523-38.

12. Park C, Park JY. Reconstruction of microtias with constricted ear features: a 22-year experience. Plast Reconstr Surg 2018;141:713-24.

13. Al-Qattan MM, Al-Omawi M. Z-plasty for Tanzer type IIb constricted ears. Ann Plast Surg 2009;63:285-7.

14. Nagata S. Alternative surgical methods of treatment for the constricted ear. Clin Plast Surg 2002;29:301-15.

15. Siegert R, Magritz R. Reconstruction of the auricle. GMS Curr Top Otorhinolaryngol Head Neck Surg 2007;6:Doc02.

16. Mohammadi AA, Imani MT, Kardeh S, et al. Non-surgical management of congenital auricular deformities. World J Plast Surg 2016;5:139-47.

17. Woo T, Kim YS, Roh TS, et al. Correction of congenital auricular deformities using the ear-molding technique. Arch Plast Surg 2016;43:512-7.

18. Ono I, Gunji H, Sato M, et al. A method of treatment of constricted ears with a conchal cartilage graft to the posterior auricular plane. Plast Reconstr Surg 1993;92:621-7.

19. Xiaogeng H, Hongxing Z, Qinghua Y, et al. A new method for correcting type I and type II constricted (cup and lop) ears. Aesthetic Plast Surg 2006;30:449-54.

20. Fisher DM, Kasrai L. Extension of autologous ear reconstruction techniques for management of the constricted ear. Paper presented at 4th International Congress on Auricular Reconstruction; October 10-12, 2007; Edinburgh, UK. 